\title{
The BGK Equation as the Limit of an N-Particle System
}

\author{
Dawan Mustafa $^{1} \cdot$ Bernt Wennberg ${ }^{2,3}$ (D)
}

Received: 3 March 2020 / Accepted: 15 June 2020 / Published online: 1 July 2020

(c) The Author(s) 2020

\section{Abstract}

The spatially homogeneous BGK equation is obtained as the limit of a model of a many particle system, similar to Mark Kac's charicature of the spatially homogeneous Boltzmann equation.

Keywords BGK equation · Particle system · Kinetic theory

Mathematics Subject Classification 35Q70 $\cdot 35 \mathrm{Q} 20 \cdot 60 \mathrm{~K} 35 \cdot 82 \mathrm{C} 22$

\section{Introduction}

The BGK equation is named after Bhatnagar, Gross, and Krook, who first presented it in an influential paper published in 1954 [3]. In its original form it is

$$
\frac{\partial f}{\partial t}+v \cdot \nabla f-\frac{e \mathbf{E}}{m} \frac{\partial f}{\partial v}=-\frac{n}{\sigma} f+\frac{n^{2}}{\sigma} \Phi
$$

Here $f=f(x, v, t)$ gives the number density of particles in phase-space $(x, v) \in \mathbb{R}^{3} \times \mathbb{R}^{3}$. The constant $\sigma>0$ controls collision rate of particles, and $\Phi=\Phi_{q, T}$ is the Maxwellian distribution

$$
\Phi(x, v, t)=\Phi_{q, T}=\frac{m}{(2 \pi k T(x, t))^{3 / 2}} \exp \left(-\frac{m}{2 k T(x, t)}(v-q(x, t))^{2}\right),
$$

Communicated by Eric A. Carlen.

Bernt Wennberg

wennberg@chalmers.se

Dawan Mustafa

dawan.mustafa@hb.se

1 University of Borås, 50190 Borås, Sweden

2 Department of Mathematical Sciences, Chalmers University of Technology, 41296 Göteborg, Sweden

3 Department of Mathematical Sciences, University of Gothenburg, 41296 Göteborg, Sweden 
where $n(x, t), q(x, t)$, and $T(x, t)$ represent the local number density, mean velocity and temperature respectively:

$$
\begin{aligned}
n(x, t) & =\int f(v, x, t) \mathrm{d} v, \\
q(x, t) & =\frac{1}{n(x, t)} \int v f(v, x, t) \mathrm{d} v, \\
\frac{3 k T(x, v)}{m} & =\frac{1}{n(x, t)} \int(v-q(x, t))^{2} f(v, x, t) \mathrm{d} v .
\end{aligned}
$$

The same kind of equation was formulated independently by Welander [25]. In [3], one considers charged particles, and $\mathbf{E}$ is the electric field computed from the particle density. It is a model of the kinetic Boltzmann equation with the purpose of providing a numerically tractable model, while retaining the most important aspects of the original Boltzmann equation: conservation of mass, momentum and energy, convergence to a unique equilibrium state, monotonicity of entropy, etc. And while easier from a computational point of view, it is considerably more difficult to analyse mathematically, and most theoretical results concerning existence and uniqueness of solutions to the BGK eqution actually hold for a modified version where the right hand side is replaced by

$$
-\frac{1}{\sigma} f+\frac{n}{\sigma} \Phi
$$

i.e. where the collision frequency is constant [20,21]. There are also results concerning solutions close to a global equilibrium, which hold also for density and temperature dependent collision frequencies $[27,28]$. There is a rather large litterature concerning various aspects of the BGK-equation dealing, for example, with methods for numerical treatment of rarefied gases (some recent examples are $[2,13,26]$ ), their fluid dynamical limits (see for example $[9,10,22]$ ), or models accounting for polyatomic gases or mixtures of different gases (for example in $[1,4,12])$, to give a few examples. A paper attempting to find a well-motivated approximation of the collision frequenecy $1 / \sigma$ can be found in [23].

The BGK equation is a fenomenological equation in the sense that it is derived explicitly to satisfy certain physical properties of a dilute gas, but until very recently there are very few published works attempting to justify the equation directly from the dynamics of an $N$-particle system. This is in contrast with the Boltzmann equation, for which there is now a rigorous derivation starting from the Liouville equation for hard sphere dynamcis, or for short range potentials, of an $N$-particle system $[11,18]$.

The BGK equation without electric field can be interpreted as a model of a large system of particles, where each particle moves independently with its own velocity. The velocity jumps at exponentially distributed intervals but remains constant in between the jumps. The jump rate is proportional to the local density of the gas, and after the jump the particle velocity is a normally distributed random variable, independent of the initial velocity, but with mean and variance determined by the local temperature and mean velocity of the gas. One can make a similar interpretation of the Boltzmann equation for hard spheres, but with two important differences. First, in (1), the collision rate only depends on the local density, and not on the velocity of the particles. In fact, Eq. (1) corresponds to a system of so-called Maxwellian molecules and not to hard spheres. The second, and more important, difference lies in the distribution of velocities of particles after a jump. The Boltzmann equation for Maxwellian molecules, which in similar notation is 


$$
\frac{\partial f}{\partial t}+v \cdot \nabla f=-\frac{n}{\sigma} f+\frac{1}{\sigma} Q^{+}(f, f)
$$

represents a process in which the velocity of a particle after the jump is given by the outcome of a random collision with a second particle drawn from the distribution with density $v \mapsto f(x, v, t) / \int_{\mathbb{R}^{3}} f(x, w, t) \mathrm{d} w$. The solutions to (5) converge to a Maxwellian distribution when $t \rightarrow \infty$, or equivalently, when the average number of velocity jumps that one particle has made, goes to infinity. In the BGK model, the velocity of a particle has a normal distribution after only one jump, and a particle system that converges to a solution of the BGK model must achieve that in the limit of infinitely many particles.

The particle system that we propose consists of particles that can have two states, active and passive, where only the active particles participate in collisions with other particles. The BGK equation will describe the evolution of passive particles in the limit of infinitely many particles. One may think of the active particles being ions, that interact at a high rate with each other, the passive ones being neutrals that do not interact. On the other hand, a neutral particle and an ion may encounter and interchange state by the transfer of an electron, so that the result is similar to allowing the velocity of a neutral particle to jump to a random velocity given by the distribution of the acitve particles. And if the collision rate for active particles is very high, then the active particles will have time to come close to an equilibrium distribution before the next exchange with the passive particles takes place.

At a formal level, one may actually pursue these ideas to derive a BGK equation of the form (1), or a hard sphere version of the same, but to make a completely rigorous derivation along the lines of for example [11] seems to be difficult [16].

An alternativ approach has been developed in [7], where an $N$-particle system is constructed in which the particles are given a normal velocity distribution after a jump, with moments computed from the empirical distributions. The authors prove rigorously that the $N$-particle model converges to the BGK model in the limit of $N$ going to infinity.

Long before a rigorous result on the validity of the Boltzmann equation had been obtained for a real particle system, Mark Kac [17] proposed a Markov jump process for the velocities of an $N$-particle distribution, and proved that in the limit as $N \rightarrow \infty$, the velocity distribution of one particle converges to the solution of a Boltzmann-like equation for a spatially homogenous gas of Maxwellian molecules with one-dimensional velocities.

In this paper we construct a Kac-type model of a system of $N$ passive and $M$ active particles, and a jump process involving collisions between active particles and the switch between active and passive state, as described above. We then prove that the one-particle distribution for passive particles converges to a BGK equation of the form

$$
\partial_{t} f(v, t)=\mathcal{M}(v, t)-f(v, t)
$$

where $\mathcal{M}$ is the standard normal distribution in one dimension. This limit can be obtained in a scaling where $M / N \rightarrow 0$ when $N \rightarrow \infty$, that is, when the fraction of active particles vanishes in the limit of infinitely many particles.

The paper is based on the results in the doctoral thesis of the first author [19]. A very similar model, with two different kinds of particles, has been presented by Bonetto et al. in [6], and also in [5]. The authors are in general interested in kinetic models coupled with a thermostat, and in the cited papers the larger set of particles ( $N$ in our paper) is considered as a thermostat acting on the smaller set of particles, and they prove that indeed, when $N \rightarrow \infty$ the large $N$-particle system is a good approximation of a Gaussian thermostat. Related results can also be found in [24].

The paper is organised as follows: In Sect. 2, we discuss Markov jump processes that give BGK-like equations in the limit of infinitely many particles, and present in full detail our 
final model. In Sect. 3 we introduce some further notation, and present the initial steps of the proof. An important step of the proof is to show that the energy partition between the passive and active particles in the limit is such that the mean energy for the active particles is one. This is proven in Sect. 4 by performing very explicit calculations of certain moments of the solutions. The proof is then concluded in Sect. 5.

\section{The Particle System, and Its Limiting Kinetic Equation}

We consider a particle system consisting of $N+M$ particles, where $N$ is number of passive particles represented by $V=\left(v_{1}, \ldots, v_{N}\right) \in \mathbb{R}^{N}$, and $M$ is number of active particles represented by $W=\left(w_{1}, \ldots, w_{M}\right) \in \mathbb{R}^{M}$. One active particle is assumed to have the same mass as one passive particle, and here that mass is set to 1 . The total kinetic energy, which thefore is $\frac{1}{2}\left(v_{1}^{2}+\cdots+v_{N}^{2}+w_{1}^{2}+\cdots+w_{M}^{2}\right)$, is assumed to be conserved, and therfore the state space of the particle system is $\mathrm{S}^{N+M-1}(\sqrt{N+M})$, the $N+M-1$-dimensional sphere of radius $\sqrt{N+M}$. Throughout the paper we also assume that $N>M$.

The dynamics of the system consist of two independent jump processes. The first one is the so-called Kac walk, which mimics the pairwise collisions of a rarefied gas. This process involves only the active particles. The second process involves a pair consisting of one active and one passive particle, and leads to an exchange of state: the passive particle becomes active, while retaining its velocity, and the active particle becomes passive. In this way there is an exchange of energy between the two sets of particles.

The Kac walk on the set of active particles is defined as follows:

- the jumps occur at exponentially distributed intervals with rate $\lambda_{2} \mathrm{NM}$.

- in a jump, a pair $\left(w_{i}, w_{j}\right)$ is is chosen uniformly among the active particles, and and $\theta \in\left[-\pi, \pi\left[\right.\right.$ is drawn from an even distribution. Then $\left(w_{i}, w_{j}\right) \mapsto\left(w_{i} \cos \theta-\right.$ $\left.w_{j} \sin \theta, w_{i} \sin \theta+w_{j} \cos \theta\right)$. With $W=\left(w_{1}, \ldots, w_{N}\right)$, this jump is denoted $W \mapsto$ $R_{i, j}(\theta) W$.

The jump rate for exchange between active and passive particles is chosen as $\lambda_{1} N$, which means that the jump rate for a given indexed passive particle $v_{j}$ is $\lambda_{1}$, independently of $N$, and that the rate at which active particles become passive is $\lambda_{1} N M^{-1}$ per active particle. Therefore the subsystem of active particles on average experience $\frac{\lambda_{2}}{\lambda_{1}} M$ jumps of Kac-type between two exchange jumps. Without loss of generality we set is $\lambda_{1}=1$, and denote the parameter $\lambda_{2}$ simply as $\lambda$ in what follows.

The intuitive picture is this: consider a time interval $\left[t_{1}, t_{2}[\right.$, where the end points are given by two consecutive exchange events. In this interval the vector $V=\left(v_{1}, \ldots, v_{N}\right)$ is unchanged, and the vector $W=\left(w_{1}, \ldots, w_{M}\right)$ will make on the order of $\lambda M N\left(t_{2}-t_{1}\right) \sim \lambda M$ steps in the Kac walk. The energy of the set of active particles is conserved by this process, and hence $|W|^{2}=w_{1}^{2}+\cdots+w_{M}^{2}$ is constant. If $\lambda$ is very large, the Kac walk will drive the distribution of $W$ to an almost uniform distribution on the sphere defined by $\left|W\left(t_{1}\right)\right|^{2}$. At $t_{2}$ a new exchange event takes place, when a randomly chosen active particle becomes passive, and hence the set of passive particles will gain a particle drawn from a distribution which is the marginal of the uniform distribution of an $M-1$-dimensional sphere. But this marginal distribution is close to a Gaussian when $M$ is large, and therefore, looking only at the distribution of passive particles, this will loose particles at exponential rate $\lambda_{1}=1$ per passive particle, and gain particles drawn from a Gaussian distribution with the same rate; this is the BGK-process for a spatially homogenous gas. 
All of this can be quantified, but some notation is needed in order to formulate a theorem. First of all we define the master equation, or forward Kolmogorov equation, corresponding to the jump process. Let $F_{N M}(V, W, t)$ be the probability density with respect to the induced measure $\sigma^{1}$ on $\mathrm{S}^{N+M-1}(\sqrt{N+M})$ for the velocities of the particles at time $t$. The time evolution of $F_{N M}$ is given by the equation:

$$
\frac{\partial}{\partial t} F_{N M}(V, W, t)=\left(L_{N M \lambda}+U_{N M}\right) F_{N M}(V, W, t),
$$

where

$$
L_{N M \lambda} F_{N M}(V, W)=\frac{2 N \lambda}{M-1} \sum_{1 \leq j<k \leq M} \int_{0}^{2 \pi}\left(F_{N M}\left(V, R_{j k}(\theta) W\right)-F_{N M}(V, W)\right) \frac{d \theta}{2 \pi},
$$

and

$$
U_{N M} F_{N M}(V, W)=\frac{1}{M} \sum_{j=1}^{N} \sum_{k=1}^{M}\left(F_{N M}\left(V_{w_{k}}^{j}, W_{v_{j}}^{k}\right)-F_{N M}(V, W)\right) .
$$

The operator $L_{N M \lambda}$ defined in (8) is the generator of the original Kac master equation acting on the $W$-variables, but with a factor $\lambda N$ in front, to give the jump rate as described above. The operator $U_{N M}$ defined in (9), with

$$
\left(V_{w_{k}}^{j}, W_{v_{j}}^{k}\right)=(\underbrace{v_{1}, \ldots, v_{j-1}, w_{k}, v_{j+1}, \ldots, v_{N}}_{V_{w_{k}}^{j}}, \underbrace{w_{1}, \ldots, w_{k-1}, v_{j}, w_{k+1}, \ldots, w_{M}}_{W_{v_{j}}^{k}})
$$

is the generator of the exchange process, when a passive and an active particle exchange their state.

An essential assumption here, just like in Kac's orginal work, is that $F_{N M}$ is symmetric with respect to permutations of the coordinates of $V$ and of the coordinates of $W$. This is to say that all passive particles are identical, and identically distributed, and that the same holds for the active particles. Hence any choice of $n$ passive particles is equivalent to choosing the first $n$. The following notation will be useful:

$$
\begin{aligned}
V_{n} & =\left(v_{1}, \ldots, v_{n}\right) \quad \text { and } \quad V^{n}=\left(v_{n+1}, \ldots, v_{N}\right) \\
W_{m} & =\left(w_{1}, \ldots, w_{m}\right) \quad \text { and } \quad W^{m}=\left(w_{m+1}, \ldots, w_{m}\right) .
\end{aligned}
$$

Definition 1 The $(n, m)$-marginals $f_{N M}^{(n m)}\left(V_{n}, W_{m}\right)$ of $F_{N M}(V, W)$ are given by the equation

$$
\begin{aligned}
& \int_{\mathrm{S}^{N+M-1}(\sqrt{N+M})} g\left(V_{n}\right) h\left(W_{m}\right) F_{N M}(V, W) d \sigma \\
= & \int_{\mathbb{R}^{n}} \int_{\mathbb{R}^{m}} g\left(V_{n}\right) h\left(W_{m}\right) f_{N M}^{(n m)}\left(V_{n}, W_{m}\right) d V_{n} d W_{m},
\end{aligned}
$$

where $g\left(V_{n}\right)$ and $h\left(W_{m}\right)$ are any bounded continuous functions on $\mathbb{R}^{n}, \mathbb{R}^{m}$, respectively, and we assume that $f_{N M}^{(n m)}$ has support in $\left\{V_{n}^{2}+W_{m}^{2} \leq N+M\right\}$.

The objective of this paper is to prove that when $N, M \rightarrow \infty$ the density of one passive particle, $f_{N M}^{(10)}(v, t)$ converges to a function $f(v, t)$ that satisfies the spatially homogeneous BGK equation (5). This is formulated in the following theorem:

1 The symbol $\sigma$ is used throughout the paper to denote the measure on the sphere $\mathrm{S}^{n-1}(r)$ induced from the Euclidian measure in $\mathbb{R}^{n}$, and therefore it is defined only in combination with the domain of integration. 
Theorem 1 Let $\left\{F_{N M}(V, W, t)\right\}_{N, M}$ be the solutions of a family of master equations (7) with $1 \leq M<N<\infty$, with initial data $F_{N M}(V, W, 0)$ satisfying

$$
\int_{\Omega_{0,0}}\left|F_{N M}(V, W, 0)\right|^{2} d \sigma(V, W) \leq C_{N M}^{2}, \int_{\Omega_{0,0}} F_{N M}(V, W, 0) v_{1}^{4} d \sigma(V, W)<\infty,
$$

$$
\frac{1}{M} \int_{\Omega_{0,0}} F_{N M}(V, W, 0)\left(\frac{1}{M} \sum_{k=1}^{M} w_{k}^{4}\right) d \sigma(V, W) \rightarrow 0 \quad \text { when } M \rightarrow \infty .
$$

Let $M=M(N), \lambda=\lambda(N)$ be such that $N / M \rightarrow \infty, N / M^{2} \rightarrow 0$ and $\lambda / C_{N M} \rightarrow \infty$ when $N \rightarrow \infty$. Then, for $0<t_{0} \leq t \leq T, \quad T<\infty$

$$
\lim _{N \rightarrow \infty} \frac{\partial}{\partial t} \int_{v_{1}^{2}<N+M} f_{N M}^{(10)}\left(v_{1}, t\right) g\left(v_{1}\right) d v_{1}=\int_{\mathbb{R}}\left(\mathcal{M}\left(v_{1}\right)-f\left(v_{1}, t\right)\right) g\left(v_{1}\right) d v_{1},
$$

where

$$
f\left(v_{1}, t\right)=\lim _{N, M \rightarrow \infty} f_{N M}^{(10)}\left(v_{1}, t\right),
$$

and where $f\left(v_{1}, t\right)$ solves the homogeneous BGK equation,

$$
\frac{\partial}{\partial t} f\left(v_{1}, t\right)=\mathcal{M}\left(v_{1}\right)-f\left(v_{1}, t\right) .
$$

So, at least weakly, the one-particle marginal of the $N+M$ dimensional particle system converges to the solution of a BGK equation, as announced in the introduction. The theorem is stated to hold uniformly for $t \geq t_{0}>0$, but to achieve convergence uniformly for all $t>0$ one must make stronger assumptions on initial data. If the initial data are chaotic, i.e. meaning that the many-particle marginals are close to products of functions of the coordinates, the $L^{2}$-norm in the theorem grows exponentially in $N+M$, and choosing $C_{N M} \sim c^{N+M}$ in Eq. (13) gives natural class of inital data for which the theorem holds.

Remark 1 An important notion in kinetic theory is that of propagation of chaos, which was made precise in Kac's paper [17]. In the present context we would say that $\left\{F_{N M}\right\}$ is a chaotic family if the marginals $\left\{f_{N M}^{(n m)}\right\}$ satisfy

$$
\lim _{N, M \rightarrow \infty} f_{N M}^{(n 0)}\left(v_{1}, \ldots, v_{n}\right)=\prod_{j=1}^{n} \lim _{N, M \rightarrow \infty} f_{N M}^{(10)}\left(v_{j}\right),
$$

and that propagation of chaos holds if the same property holds for all times provided it holds initially. Here only marginals with respect to the $v$ variables are included because the limiting equation only involves the distribution of passive particles. These do not interact directly, but jump almost independently. When $N$ and $M$ are bounded, some correlation is created because each jump of a passive particle changes the distribution of the active particles, the effect of this vanishes when the number of active particles, $M$, increase to infinity. A more rigorous statement can be made from the observation that the proof of Theorem 1 with very small changes shows that Eq. (14) also holds for the marignals $f_{N M}^{(20)}$, whith the Maxwellian $\mathcal{M}\left(v_{1}\right)$ replaced by a bivariate Maxwellian $\mathcal{M}\left(v_{1}, v_{2}\right)$, which itself factorizes. 


\section{Initial Steps of the Proof}

To prepare for the proof of Theorem 1, we first present a few well-known formulae concerning spheres. A first observation is that although the $V$ - and $W$-variables represent particles in different states, they behave exactly as variables for integration over the sphere $\Omega_{0,0}=$ $\left\{|V|^{2}+|W|^{2}=N+M\right\}$, and therefore, for any function $G(V, W)$, we have

$$
\int_{\Omega_{0,0}} G\left(V_{w_{k}}^{j}, W_{v_{j}}^{k}\right) d \sigma(V, W)=\int_{\Omega_{0,0}} G(V, W) d \sigma(V, W) .
$$

The area of an $n-1$ dimensional sphere of radius $r$,

$\mathrm{S}^{n-1}(r)=\left\{\left(x_{1}, \ldots, x_{n}\right) \in \mathbb{R}^{n} \mid x_{1}^{2}+x_{2}^{2} \cdots+x_{n}^{2}=r^{2}\right\}$ is given by

$$
\left|\mathrm{S}^{n-1}(r)\right|=r^{n-1} \frac{2 \pi^{\frac{n}{2}}}{\Gamma\left(\frac{n}{2}\right)}=r^{n-1}\left|\mathrm{~S}^{n-1}\right| .
$$

For any function $f$ defined on $\mathrm{S}^{n-1}(r)$ one may write

$$
\begin{aligned}
& \int_{\mathrm{S}^{n-1}(r)} f\left(x_{1}, \ldots, x_{n}\right) d \sigma\left(x_{1}, \ldots, x_{n}\right) \\
= & \int_{x_{1}^{2}+\cdots+x_{k}^{2} \leq r^{2}}\left(\frac{r^{2}}{r^{2}-x_{1}^{2}-\cdots-x_{k}^{2}}\right)^{1 / 2} \\
& \int_{\mathrm{S}^{n-k-1}\left(\sqrt{r^{2}-x_{1}^{2}-\cdots-x_{k}^{2}}\right)} f\left(x_{1}, \ldots, x_{n}\right) d \sigma\left(x_{k+1}, \ldots, x_{n}\right) d x_{1} d x_{2} \ldots d x_{k} .
\end{aligned}
$$

Therefore the marginals defined in Definition 1 may be written explicitly as

$$
f_{N M}^{(n m)}\left(V_{n}, W_{m}\right)=\Gamma_{n, m} \int_{\Omega_{n, m}} F_{N M}(V, W) d \sigma\left(V^{n}, W^{m}\right),
$$

where we introduce the notation

$$
\begin{aligned}
\Gamma_{n, m} & =\frac{(N+M)^{1 / 2}}{\left(N+M-\left|V_{n}\right|^{2}-\left|W_{m}\right|^{2}\right)^{1 / 2}}, \quad \text { and } \\
\Omega_{n, m} & =\mathrm{S}^{N+M-n-m-1}\left(\sqrt{N+M-\left|V_{n}\right|^{2}-\left|W_{m}\right|^{2}}\right) .
\end{aligned}
$$

We also define the average with respect to the $W$-variables as follows:

Definition 2 Let $F_{N M}(V, W) \in L^{1}\left(\mathrm{~S}^{N+M-1}(r)\right)$. Then

$$
\bar{F}_{N M}(V)=\left|\mathrm{S}^{M-1}\left(\sqrt{r^{2}-|V|^{2}}\right)\right|_{\mathrm{S}^{M-1}\left(\sqrt{r^{2}-|V|^{2}}\right)}^{-1} F_{N M}(V, W) d \sigma(W) .
$$

Finally we compute the marginal of the first coordinate of a point chosen uniformly on an $M$-dimensional sphere of radius $\sqrt{M}$. The uniform density is given by the constant function 
$\left|\mathrm{S}^{M-1}\right|^{-1} M^{-(M-1) / 2}$, and hence the marginal of the first coordinate is

$$
\begin{aligned}
\mathcal{M}_{M}\left(x_{1}\right)= & \left(\frac{M}{M-x_{1}^{2}}\right)^{1 / 2} \int_{\mathrm{S}^{M-2}\left(\sqrt{M-x_{1}^{2}}\right)}\left|\mathrm{S}^{M-1}\right|^{-1} M^{-(M-1) / 2} d \sigma\left(x_{2}, \ldots, x_{M}\right) \\
= & \left(\frac{M}{M-x_{1}^{2}}\right)^{1 / 2} \frac{\left|\mathrm{S}^{M-2}\right|}{\left|\mathrm{S}^{M-1}\right|} \frac{\left(M-x_{1}^{2}\right)^{(M-2) / 2}}{M^{(M-1) / 2}}=\frac{1}{\sqrt{M}} \frac{\left|\mathrm{S}^{M-2}\right|}{\left|\mathrm{S}^{M-1}\right|}\left(1-\frac{x_{1}^{2}}{M}\right)^{(M-3) / 2} \\
& \underset{M \rightarrow \infty}{\longrightarrow} \frac{1}{\sqrt{2 \pi}} e^{-x_{1}^{2} / 2}=\mathcal{M}\left(x_{1}\right) .
\end{aligned}
$$

The first step in our proof of Theorem 1 is to integrate over the $W$-variables in Eq. (7), to find an evolution equation for the $V$-marginal of $F_{N, M}$. It is

$$
\begin{aligned}
& \frac{\partial}{\partial t} f_{N M}^{(N 0)}(V, t) \\
& \quad=\Gamma_{N, 0} \int_{\Omega_{N, 0}}\left(L_{N M \lambda}+U_{N M}\right) F_{N M}(V, W, t) d \sigma(W) \\
& \quad=\sum_{j=1}^{N} \Gamma_{N, 0} \int_{\Omega_{N, 0}}\left(F_{N M}\left(V_{w_{1}}^{j}, W_{v_{j}}^{1}, t\right)-F_{N M}(V, W, t)\right) d \sigma(W) .
\end{aligned}
$$

The integral of $L_{N M \lambda} F_{N M}(V, W, t)$ vanishes because the generator of the Kac walk conserves mass, and we also use the symmetry with respect to permutations of the $W$-coordinates to replace the sum over $k$ in (9) with $M$ terms, all involving $w_{1}$. Adding and subtracting $\bar{F}_{N M}\left(V_{w_{1}}^{j}\right)$ we obtain

$$
\begin{aligned}
\frac{\partial}{\partial t} f_{N M}^{(N 0)}(V, t) & \\
= & \sum_{j=1}^{N} \Gamma_{N, 0} \int_{\Omega_{N, 0}}\left(\bar{F}_{N M}\left(V_{w_{1}}^{j}, t\right)-F_{N M}(V, W, t)\right) d \sigma(W) \\
& +\sum_{j=1}^{N} \Gamma_{N, 0} \int_{\Omega_{N, 0}}\left(F_{N M}\left(V_{w_{1}}^{j}, W_{v_{j}}^{1}, t\right)-\bar{F}_{N M}\left(V_{w_{1}}^{j}, t\right)\right) d \sigma(W) \\
= & I_{1}(V)+I_{2}(V) .
\end{aligned}
$$

We will show that by a suitable choice of $\lambda$, which is hidden here because it only affects the Kac operator $L_{N, M, \lambda}$, the term $I_{2}(V)$ vanishes in the limit, and hence that the evolution of $f_{N M}^{(N 0)}(V)$ essentially is governed by $I_{1}(V)$, which in turn will reproduce the righthand side of the BGK equation in the limit when $N, M \rightarrow \infty$.

We denote the $N$ terms of $I_{1}(V)$ as $I_{1, j}(V)$, so that $I_{1}(V)=\sum_{j=1}^{N} I_{1, j}(V)$. For $j \neq 1$, and for any function $g \in C(\mathbb{R})$, 


$$
\begin{aligned}
& \int_{|V|^{2} \leq N+M} g\left(v_{1}\right) I_{1, j}(V) d V \\
= & \int_{|V|^{2}+|W|^{2}=N+M} g\left(v_{1}\right)\left(\bar{F}_{N M}\left(V_{w_{j}^{1}}^{j}, t\right)-\bar{F}_{N M}(V, t)\right) d \sigma(V, W)=0,
\end{aligned}
$$

by the argument in Eq. (18), and therefore it is sufficient to consider the first term, $I_{1,1}(V)$. Using Eq. (21) and Definition 2, the integral in this term is

$$
\begin{aligned}
& \int_{\Omega_{N, 0}} \bar{F}_{N M}\left(V_{w_{1}}^{1}, t\right) d \sigma(W)-\left|\Omega_{N, 0}\right| \bar{F}_{N M}(V, t) \\
& \quad=\left|\Omega_{N, 0}\right| \frac{1}{\mathrm{~S}^{M-1}(\sqrt{M})} \int_{\mathrm{S}^{M-1}(\sqrt{M})} \bar{F}_{N M}\left(V_{\tilde{w}_{1} \sqrt{\tau(V)}}^{1}, t\right) d \sigma(\widetilde{W})-\left|\Omega_{N, 0}\right| \bar{F}_{N M}(V, t),
\end{aligned}
$$

where we have made the change of variables $W \mapsto \sqrt{\tau(V)} \tilde{W}$, with

$$
\tau(V)=\frac{N+M-|V|^{2}}{M},
$$

the average energy per active particle. The integral is then the marginal distribution of the uniform density over an $M-1$-dimensional sphere, as in Eq. (24), so that finally the $I_{1,1}(V)$ becomes

$$
\begin{gathered}
\Gamma_{N, 0}\left|\Omega_{N, 0}\right| \int_{-\sqrt{M}}^{\sqrt{M}} \bar{F}_{N M}\left(V_{w \sqrt{\tau(V)}}^{1}, t\right) \Psi_{M}(w) d w-\Gamma_{N, 0}\left|\Omega_{N, 0}\right| \bar{F}_{N M}(V, t) \\
=\int_{-\sqrt{N+M-|V|^{2}}} \frac{\Psi_{M}\left(v_{1} / \sqrt{\tau\left(V_{w_{1}}^{1}\right)}\right)}{\sqrt{\tau\left(V_{w_{1}}^{1}\right)}} f_{N M}^{(N 0)}\left(V_{w_{1}}^{1}, t\right) d w_{1}-f_{N M}^{(N 0)}(V, t) .
\end{gathered}
$$

This integral can now be written as a sum of three terms as follows:

$$
\begin{aligned}
& \sqrt{N+M-|V|^{2}} \\
& \int_{-\sqrt{N+M-|V|^{2}}} \mathcal{M}\left(v_{1}\right) f_{N M}^{(N 0)}\left(V_{w_{1}}^{1}, t\right) d w_{1}-f_{N M}^{(N 0)}(V, t) \\
& +\int_{-\sqrt{N+M-|V|^{2}}}^{\sqrt{N+M-|V|^{2}}}\left(\mathcal{M}_{M}\left(v_{1}\right)-\mathcal{M}\left(v_{1}\right)\right) f_{N M}^{(N 0)}\left(V_{w_{1}}^{1}, t\right) d w_{1} \\
& +\int_{-\sqrt{N+M-|V|^{2}}}^{\sqrt{N+M-|V|^{2}}}\left(\frac{\mathcal{M}_{M}\left(v_{1} / \sqrt{\tau\left(V_{w_{1}}^{1}\right)}\right)}{\sqrt{\tau\left(V_{w_{1}}^{1}\right)}}-\mathcal{M}_{M}\left(v_{1}\right)\right) f_{N M}^{(N 0)}\left(V_{w_{1}}^{1}, t\right) d w_{1} \\
& :=a(V, t)+b(V, t)+c(V, t) \text {. }
\end{aligned}
$$


Consider the first of these terms, $a(V, t)$. For arbitrary $g(v) \in C(\mathbb{R})$,

$$
\int_{|V|^{2} \leq N+M} a(V, t) g\left(v_{1}\right) d V=\int_{v_{1}^{2} \leq N+M}\left(\mathcal{M}\left(v_{1}\right)-f_{N M}^{(10)}\left(v_{1}, t\right)\right) g\left(v_{1}\right) d v_{1},
$$

which converges to the right-hand side of equation (14) in Theorem 1 . Therefore the proof can be concluded by proving that the other terms vanish.

The second term, an integral of $b(V, t)$, converges to zero, because

$$
\begin{aligned}
& \int_{|V|^{2} \leq N+M} g\left(v_{1}\right) b(V, t) d V \\
= & \int_{|V|^{2}+w_{1}^{2} \leq N+M} g\left(v_{1}\right)\left(\mathcal{M}_{M}\left(v_{1}\right)-\mathcal{M}\left(v_{1}\right)\right) f_{N M}^{(N 0)}\left(V_{w_{1}}^{1}, t\right) d w_{1} d V \\
= & \int_{|V|^{2} \leq N+M} \int_{-\sqrt{N+M-|V|^{2}}}^{\sqrt{N+M-|V|^{2}}}\left(\mathcal{M}_{M}(u)-\mathcal{M}(u)\right) g(u) d u f_{N M}^{(N 0)}(V, t) d V,
\end{aligned}
$$

and we know that the $\mathcal{M}_{M}(w) \rightarrow \mathcal{M}(w)$ pointwise, when $M \rightarrow \infty$.

Of the three terms $a(V, t), b(V, t)$, and $c(V, t)$, the last one is the most difficult to analyse. This is the subject of Sect. 4, where it is proven that on the domain of integration, we have $\tau(V) \rightarrow 1$ when $N, M \rightarrow \infty$ under the constraints given in Theorem 1 .

The proof of Theorem 1 can be concluded with these three estimates, together with a proof that $\int g\left(v_{1}\right) I_{2}(V) d v \rightarrow 0$ when $N, M \rightarrow \infty$. The remainig part of this section is devoted to proving that $I_{2}(V)$ converges to zero with suitable choices of $N, M$ and $\lambda$. The result is largely due to Lemma 1 below, which in turn follows from a result on the spectral gap for the generator $L_{N M \lambda}$ of the Kac walk. Without the operator $U_{N M}$, the generator for the exchange between passive and active particles, Eq. (7) becomes

$$
\frac{\partial}{\partial t} F_{N M}(V, W, t)=L_{N M \lambda} F_{N M}(V, W, t),
$$

which is the original Kac master equation in the $M$ variables $\left(w_{1}, \ldots, w_{M}\right)$, with the $V$ variables appearing only as parameters. Kac conjectured that the spectral gap $\Delta_{M}$ of $-\mathcal{L}_{M}=$ $-(\lambda N)^{-1} L_{N M \lambda}$ is bounded away from 0 , uniformly in the number of particles $M$. The parameter $V$ is of course not present i Kac's work, but it doesn't have an influence on the spectral gap, because this gap does not depend on the total energy of the system, and $\lambda N$ only serves to increase the jump rate. Kac's conjecture was first proved by Janvresse [15], and an exact formula for the gap of $-\mathcal{L}_{M}$ was later obtained by Carlen et al. [8]:

$$
\Delta_{M}=\frac{1}{2} \frac{M+2}{M-1} \geq \frac{1}{2}
$$

and the corresponding eigenfunction is

$$
\phi_{\Delta_{M}}=\sum_{j=1}^{M}\left(w_{j}^{4}-\frac{3 M}{M+2}\left(\frac{r^{2}}{M}\right)^{2}\right),
$$

where $r$ is the radius of the $M-1$-dimensional sphere. In fact, this eigenvalue and eigenfunction were computed also in [14], but without a proof that this is also determines the spectral gap. 
It follows that in an interval $t_{1}<t<t_{2}$ defined by two consecutive exchange events,

$$
\left\|F_{N M}(V, \cdot, t)-\bar{F}_{N M}(V, t)\right\|_{2} \leq e^{-\frac{N \lambda}{2}\left(t-t_{1}\right)}\left\|F_{N M}\left(V, \cdot t_{1}\right)-\bar{F}_{N M}\left(V, t_{1}\right)\right\|_{2} .
$$

With $\lambda$ very large, the term $U_{N M} F$ can be considered to be a small perturbation, which is expressed in the following lemma:

Lemma 1 Let $F_{N M}(V, W, t)$ be a solution to Eq. (7), and let $\bar{F}_{N M}(V, t)$ be defined by Definition 2. Then, for all $t \geq 0$ and $V \in \mathbb{R}^{N}$,

$$
\begin{aligned}
& \left\|F_{N M}(V, \cdot, t)-\bar{F}_{N M}(V, t)\right\|_{2} \\
& \quad \leq e^{-\frac{t N \lambda}{2}}\left\|F_{N M}(V, \cdot, 0)-\bar{F}_{N M}(V, 0)\right\|_{2} \\
& \quad+\frac{1-e^{-\frac{t N \lambda}{2}}}{2 N \lambda} \sup _{0 \leq s \leq t}\left\|U_{N M} F_{N M}(V, \cdot, s)-\overline{U_{N M} F}(V, s)\right\|_{2},
\end{aligned}
$$

where the norm is in $L^{2}\left(\Omega_{N, 0}, d \sigma(W)\right)$.

Proof By the Duhamel formula we can write

$$
F_{N M}(V, W, t)=e^{t L_{N M \lambda}} F_{N M}(V, W, 0)+\int_{0}^{t} e^{(t-s) L_{N M \lambda}} U_{N M} F_{N M}(V, W, s) d s .
$$

Because $e^{t L_{N M \lambda}}$ acts only in the $W$ variables, and conserves mass and leaves the uniform density on the sphere invariant, an integration over $\Omega_{N, 0}$ gives

$$
\begin{aligned}
\bar{F}_{N M}(V, t)= & e^{t L_{N M \lambda}} \bar{F}_{N M}(V, 0) \\
& +\int_{0}^{t} e^{(t-s) L_{N M \lambda}} \frac{1}{\left|\Omega_{N, 0}\right|} \int_{\Omega_{N, 0}} U_{N M} F_{N M}(V, W, s) d \sigma(W) d s,
\end{aligned}
$$

and so

$$
\begin{aligned}
& F_{N M}(V, W, t)-\bar{F}_{N M}(V, t) \\
& =e^{t L_{N M \lambda}}\left(F_{N M}(V, W, 0)-\bar{F}_{N M}(V, 0)\right) \\
& \quad+\int_{0}^{t} e^{(t-s) L_{N M \lambda}}\left(U_{N M} F_{N M}(V, W, s)-\overline{U_{N M} F}(V, W, s)\right) d s .
\end{aligned}
$$

The formula for the spectral gap for the Kac model yields

$$
\begin{aligned}
& \left\|F_{N M}(V, W, t)-\bar{F}_{N M}(V, t)\right\|_{L^{2}\left(\mathrm{~S}^{M-1}\left(\sqrt{N+M-|V|^{2}}\right), d \sigma\right)} \\
& \quad \leq e^{-\frac{t N \lambda}{2}}\left\|F_{N M}(V, W, 0)-\bar{F}_{N M}(V, 0)\right\|_{\left.L^{2}\left(\Omega_{N, 0}\right), d \sigma\right)} \\
& \quad+\int_{0}^{t} e^{-\frac{(t-s) N \lambda}{2}}\left\|U_{N M} F_{N M}(V, W, s)-\overline{U_{N M} F}(V, W, s)\right\|_{L^{2}\left(\mathrm{~S}^{M-1}\left(\Omega_{N, 0}\right), d \sigma\right)} d s .
\end{aligned}
$$

A simple computation concludes the proof.

The desired estimate of $I_{2}(V)$ is a direct consequence of Lemma 1:

Lemma 2 Assume that $F_{N M}(V, W, 0) \in L^{2}\left(\Omega_{0,0}, d \sigma(V, W)\right)$. Let $F_{N M}(V, W, t)$ be the solution of $E q$. (7) and $\bar{F}_{N M}(V, t)$ be given by Definition 2 (Eq. (23)). Then, for every bounded function $g: \mathbb{R} \rightarrow \mathbb{R}$ and all $t \geq 0$

$$
\left|\int_{|V|^{2} \leq N+M} I_{2}(V) g\left(v_{1}\right) d V\right|^{2} \leq\left(2 e^{-\lambda N t}+\frac{4}{\lambda^{2}}\right)\|g\|_{\infty}^{2}\left\|F_{N M}(\cdot, \cdot, 0)\right\|_{L^{2}\left(\Omega_{0,0}\right)}^{2}
$$


Proof Multiplying Eq. (7) by $F_{N M}(V, W, t)$ and integrating over $\Omega_{0,0}$ with respect to $\sigma(V, W)$, and using that $L_{M N \lambda}$ is a non-positive operator, it follows that

$$
\frac{d}{d t} \int_{\Omega_{0,0}}\left|F_{N M}(V, W, t)\right|^{2} d \sigma(V, W) \leq \int_{\Omega_{0,0}} F_{N M}(V, W, t) U_{N M} F_{N M}(V, W, t) d \sigma(V, W) .
$$

According to the definition of $U_{N M}$ (Eq. (9)), the righthand side of this expression is a sum of terms of the form $F_{N M}(V, W, t)\left(F_{N M}\left(V_{w_{k}}^{j}, W_{v_{j}}^{k}, t\right)-F_{N M}(V, W, t)\right)$, which due to the inequality $2 a b \leq a^{2}+b^{2}$ is smaller than or equal to $\frac{1}{2}\left(F_{N M}\left(V_{w_{k}}^{j}, W_{v_{j}}^{k}, t\right)^{2}-F_{N M}(V, W, t)^{2}\right)$.

After integration all these terms give a non-positive contribution, and hence the $L^{2}$-norm of $F$ is non increasing. Therefore the righthand side of the inequality (39) is non-positive, and we have

$$
\int_{\Omega_{0,0}}\left|F_{N M}(V, W, t)\right|^{2} d \sigma(V, W) \leq \int_{\Omega_{0,0}}\left|F_{N M}(V, W, 0)\right|^{2} d \sigma(V, W),
$$

and $F_{N M}(V, W, t) \in L^{2}\left(\Omega_{0,0}\right)$ for all $t \geq 0$. The function $I_{2}(V)$ is defined as the second sum in the right hand side of equation (26). When multiplying that expression with a function $g\left(v_{1}\right)$ (a function depending only on one variable), only the term with $j=1$ remains, and therefore

$$
\begin{aligned}
\int_{|V|^{2} \leq N+M} I_{2}(V) g\left(v_{1}\right) d V & =\int_{|V|^{2} \leq N+M} \Gamma_{N, 0} g\left(v_{1}\right) \int_{\Omega_{N, 0}}\left(F_{N M}\left(V_{w_{1}}^{1}, W_{w_{1}}^{1}, t\right)\right. \\
& \left.-\bar{F}_{N M}\left(V_{w_{1}}^{1}, t\right)\right) d \sigma(W) d V \\
& =\int_{\Omega_{0,0}} g\left(v_{1}\right)\left(F_{N M}\left(V_{w_{1}}^{1}, W_{w_{1}}^{1}, t\right)-\bar{F}_{N M}\left(V_{w_{1}}^{1}, t\right)\right) d \sigma(V, W) .
\end{aligned}
$$

Using the Cauchy-Schwartz inequality, and Lemma 1 we get

$$
\begin{aligned}
& \left|\int_{|V|^{2} \leq N+M} I_{2}(V) g\left(v_{1}\right) d V\right|^{2} \leq\|g\|_{\infty}^{2} \int_{|V|^{2} \leq N+M} \Gamma_{N, 0} \int_{\Omega_{N, 0}}\left|F_{N M}(V, \cdot, t)-\bar{F}_{N M}(V, t)\right|^{2} d \sigma(W) d V \\
& \leq 2\|g\|_{\infty}^{2} e^{-\lambda N t} \int_{|V|^{2} \leq N+M} \Gamma_{N, 0} \int_{\Omega_{N, 0}}\left|F_{N M}(V, \cdot, 0)-\bar{F}_{N M}(V, 0)\right|^{2} d \sigma(W) d V \\
& +\frac{2\|g\|_{\infty}^{2}}{N^{2} \lambda^{2}} \int_{|V|^{2} \leq N+M} \Gamma_{N, 0} \sup _{0 \leq s \leq t} \int_{\Omega_{N, 0}}\left|U_{N M} F_{N M}(V, \cdot, s)-\overline{U_{N M} F}(V, s)\right|^{2} d \sigma(W) d V .
\end{aligned}
$$

A calculation using (9) shows that

$$
\int_{\Omega_{0,0}}\left|U_{N M} F_{N M}(V, \cdot, s)-\overline{U_{N M} F}(V, s)\right|^{2} d \sigma(W) d V \leq 2 N^{2} \int_{\Omega_{0,0}}\left|F_{N M}(V, W, s)\right|^{2} d \sigma(V, W),
$$

and so, collecting all the inequalities we finally obtain the inequality (38), which concludes the proof. 


\section{Evolution of Moments and Energy Partition}

The average energy per active particle is

$\int_{\Omega_{0,0}} F_{N M}(V, W) \frac{1}{M}|W|^{2} d \sigma(V, W)=\int_{\Omega_{0,0}} F_{N M}(V, W) \frac{1}{M}\left(N+M-|V|^{2}\right) d \sigma(V, W)$

and the main purpose with this section is to prove that, for large $N, M$, the density $F_{N M}$ is concentrated near the set $\left\{\left.(V, W) \in \Omega_{0,0}\left|\frac{1}{M}\right| W\right|^{2}=1\right\}$. The proof goes by estimates of moments of the form

$$
\int_{\Omega_{0,0}} F_{N M}(V, W) H(V) d \sigma(V, W) \text { or } \quad \int_{\Omega_{0,0}} F_{N M}(V, W) H(W) d \sigma(V, W),
$$

one of them being

$$
\psi(t)=\int_{\Omega_{0,0}} F_{N M}(V, W)\left(\frac{1}{M}|W|^{2}-1\right)^{2} d \sigma(V, W)
$$

We prove that for all $t>0, \psi(t) \rightarrow 0$ when $N, M, \lambda \rightarrow \infty$ in a suitable way.

The starting point is Eq. (35),

$$
F_{t}=e^{t L} F_{0}+\int_{0}^{t} e^{(t-s) L} U F_{s} d s
$$

where, simplifying notation, $F_{t}=F_{N M}(V, W, t)$, and the operators $L=L_{N, M, \lambda}$, and $U=U_{N, M}$ are given in Eqs. (8) and (9). The two operators $U$ and $L$ are self adjoint, but they don't commute. Multiplying the terms in (44) with $H(V)$ and integrating gives

$$
\begin{aligned}
& \int_{\Omega_{0,0}} F_{t}(V, W) H(V) d \sigma \\
& =\int_{\Omega_{0,0}}\left(e^{t L} F_{0}(V, W)\right) H(V) d \sigma+\int_{0}^{t} \int_{\Omega_{0,0}}\left(e^{(t-s) L}\left[U F_{s}\right](V, W)\right) H(V) d \sigma d s \\
& =\int_{\Omega_{0,0}} F_{0}(V, W) e^{t L} H(V) d \sigma+\int_{0}^{t} \int_{\Omega_{0,0}}\left[U F_{s}\right](V, W) e^{(t-s) L} H(V) d \sigma d s \\
& =\int_{\Omega_{0,0}} F_{0}(V, W) H(V) d \sigma+\int_{0}^{t} \int_{\Omega_{0,0}}\left[U F_{S}\right](V, W) H(V) d \sigma d s \\
& =\int_{\Omega_{0,0}} F_{0}(V, W) H(V) d \sigma+\int_{0}^{t} \int_{\Omega_{0,0}} F_{S}(V, W)[U H](V, W) d \sigma d s
\end{aligned}
$$

because $L$ acts only in the $W$-variables, and constants are left invariant by $L$. 
The calculations for moments of the form $H(W)$, are much simplified when $H(W)$ is an eigenfunction of the operator $L$ with eigenvalue $\lambda_{H}$. In this case

$$
\begin{aligned}
& \int_{\Omega_{0,0}} F_{t}(V, W) H(W) d \sigma \\
& \quad=\int_{\Omega_{0,0}} F_{0}(V, W) e^{t L} H(W) d \sigma+\int_{0}^{t} \int_{\Omega_{0,0}}\left[U F_{s}\right](V, W) e^{(t-s) L} H(W) d \sigma d s \\
& =e^{\lambda_{H} t} \int_{\Omega_{0,0}} F_{0}(V, W) H(W) d \sigma+\int_{0}^{t} e^{(t-s) \lambda_{H}} \int_{\Omega_{0,0}} F_{S}(V, W)[U H](V, W) d \sigma d s .
\end{aligned}
$$

In all the integrals we need to compute $[U H](V, W)$, where the functions $H(V)$ and $H(W)$ are given by expressions of the form

$$
\begin{aligned}
& H(V)=\frac{1}{N} \sum_{j=1}^{N} h\left(v_{j}\right), \text { or } \\
& H(V)=\Phi\left(|V|^{2}\right),
\end{aligned}
$$

or combinations of these, and similar with functions depending only on $W$. Then $[U H](V, W)$ is given by the sum in (9) as

$$
\frac{1}{M} \sum_{j=1}^{N} \sum_{k=1}^{M}\left(H\left(V_{w_{k}}^{j}\right)-H(V)\right) \text {, or } \frac{1}{M} \sum_{j=1}^{N} \sum_{k=1}^{M}\left(H\left(W_{v_{j}}^{k}\right)-H(W)\right) .
$$

When $H(V)$ and $H(W)$ are of the form (47), then

$$
H\left(V_{w_{k}}^{j}\right)=H(V)+\frac{1}{N}\left(h\left(w_{k}\right)-h\left(v_{j}\right)\right), \text { and } \quad H\left(V_{v_{k}}^{j}\right)=\Phi\left(|V|^{2}+w_{k}^{2}-v_{j}^{2}\right)
$$

respectively.

The mean energy per active particle in a given configuration $(V, W)$ is

$$
\tau(V)=\frac{|W|^{2}}{M}=\frac{1}{M}\left(N+M-|V|^{2}\right)=1+\frac{N-|V|^{2}}{M},
$$

and in addition to $\tau(V)$ we introduce the notation

$$
m_{4}(W)=\frac{1}{M} \sum_{k=1}^{M} w_{k}^{4} \quad \text { and } \quad m_{4}(V)=\frac{1}{N} \sum_{j=1}^{N} v_{j}^{4},
$$

as well as

$$
\tilde{m}_{4}(V)=\frac{1}{\left|\Omega_{N, 0}\right|} \int_{\Omega_{N, 0}} m_{4}(W) d \sigma(W)
$$

the average of $m_{4}(W)$ over the sphere $\Omega_{N, 0}$. This average can be expressed in terms of the radius of $\Omega_{N, 0},|W|$, which in turn is a function of $|V|$. We have

$$
\begin{aligned}
\tilde{m}_{4}(V) & =\frac{3 M}{M+2}\left(\frac{|W|^{2}}{M}\right)^{2}=\frac{3 M}{M+2}\left(\frac{N+M-|V|^{2}}{M}\right)^{2} \\
& =\frac{3 M}{M+2}\left((\tau(V)-1)^{2}+2(\tau(V)-1)+1\right) .
\end{aligned}
$$


Using this notation, we define the following moments:

$$
\begin{aligned}
\eta(t) & =\int_{\Omega_{0,0}} F_{N M}(V, W, t)(\tau(V)-1) d \sigma \\
\psi(t) & =\int_{\Omega_{0,0}} F_{N M}(V, W, t)(\tau(V)-1)^{2} d \sigma, \quad \text { (already defined in Eq. (43)) } \\
\zeta(t) & =\int_{\Omega_{0,0}} F_{N M}(V, W, t)\left(m_{4}(W)-\tilde{m}_{4}(V)\right) d \sigma, \quad \text { and } \\
\xi(t) & =\int_{\Omega_{0,0}} F_{N M}(V, W, t) m_{4}(V) d \sigma .
\end{aligned}
$$

The expression $m_{4}(W)-\tilde{m}_{4}(V)$ is the eigenfunction of the operator $L$ corresponding to the eigenvalue $\lambda N \Delta_{M}$. We now obtain expressions for these moments using the Eqs. (45) and $(46)^{2}$

First setting $H(V)=\tau(V)-1=\frac{N-|V|^{2}}{M}$ gives

$$
\begin{aligned}
-\frac{N}{M}\left(\frac{1}{M}|W|^{2}-\frac{1}{N}|V|^{2}\right) & =-\frac{N}{M}\left(\frac{1}{M}\left(N+M-|V|^{2}\right)-\frac{1}{N}|V|^{2}\right) \\
& =-\left(1+\frac{N}{M}\right)(\tau(V)-1),
\end{aligned}
$$

and therefore, with $v_{N, M}=1+N / M$,

$$
\eta(t)=\eta(0)-v_{N, M} \int_{s=0}^{t} \eta(s) d s
$$

which means that the mean energy per active particle converges exponentially to 1 as $N / M \rightarrow$ $\infty$.

Similarly, to find an expression for $\psi(t)$, we take $H(V)=(\tau(V)-1)^{2}=\left(\frac{N-|V|^{2}}{M}\right)^{2}$ and obtain the expression

$$
\begin{aligned}
H\left(V_{w_{k}}^{j}\right)-H(V) & =\left(\frac{N-|V|^{2}+v_{j}^{2}-w_{k}^{2}}{M}\right)^{2}-\left(\frac{N-|V|^{2}}{M}\right)^{2} \\
& =\frac{2}{M}\left(\frac{N-|V|^{2}}{M}\right)\left(v_{j}^{2}-w_{k}^{2}\right)+\left(\frac{v_{j}^{2}-w_{k}^{2}}{M}\right)^{2} .
\end{aligned}
$$

Using

$$
\begin{aligned}
\frac{1}{M} \sum_{j, k}\left(v_{j}^{2}-w_{k}^{2}\right) & =-v_{N, M} M(\tau(V)-1) \quad \text { and } \\
\frac{1}{M} \sum_{j, k} v_{j}^{2} w_{k}^{2} & =\frac{1}{M}|V|^{2}|W|^{2}=-M(\tau(V)-1)^{2}+(N-M)(\tau(V)-1)+N
\end{aligned}
$$

2 Some of the calculations are rather messy, and we have used a computer algebra system for checking these calulations as well as for analysing the linear system for the moments. The code showing all operations are available upon request from the corresponding author. 
and evaluating the sum in (48) gives

$$
\begin{aligned}
{[U H](V, W)=} & -2\left(v_{N, M}-\frac{1}{M}\right)(\tau(V)-1)^{2}-\left(\frac{2 N}{M^{2}}-\frac{2}{M}\right)(\tau(V)-1)-\frac{2 N}{M^{2}} \\
& +\frac{N}{M^{2}}\left(m_{4}(V)+m_{4}(W)\right) .
\end{aligned}
$$

Because

$$
m_{4}(W)=m_{4}(W)-\tilde{m}_{4}(V)+\frac{3 M}{2+M}\left((\tau(V)-1)^{2}+2(\tau(V)-1)+1\right)
$$

it follows that $\psi(t)$ satisfies

$$
\begin{aligned}
\psi(t)= & \psi(0)+\int_{0}^{t}\left(-2\left(v_{N, M}-\frac{1}{M}\right) \psi(s)-\left(\frac{2 N}{M^{2}}-\frac{2}{M}\right) \eta(s)-\frac{2 N}{M^{2}}\right) d s \\
& +\int_{0}^{t} \frac{N}{M^{2}}\left(\xi(s)+\zeta(s)+\frac{3 M}{M+2}(\psi(s)+2 \eta(s)+1)\right) d s .
\end{aligned}
$$

Next, taking $H(V)=m_{4}(V)$ in (45) gives an expression for $\xi(t)$. With

$$
[U H](V, W)=m_{4}(W)-m_{4}(V)=-m_{4}(V)+\left(m_{4}(W)-\bar{m}_{4}(V)\right)+\frac{3 M}{M+2} \tau(V)^{2},
$$

and integrating over $V$ and $W$ results in

$$
\begin{aligned}
\xi(t)= & \xi(0) \\
& +\int_{0}^{t}\left(-\xi(s)+\zeta(s)+\frac{3 M}{M+2}(\psi(s)+2 \eta(s)+1)\right) d s .
\end{aligned}
$$

And finally, with $H(W)=m_{4}(W)-\tilde{m}_{4}(V)=\frac{1}{M} \sum_{k=1}^{M} w_{k}^{4}-\frac{3}{M(M+2)}|W|^{4}$, we get

$$
\begin{aligned}
H\left(W_{v_{j}}^{k}\right)-H(W)= & \frac{1}{M}\left(v_{j}^{4}-w_{k}^{4}\right) \\
& -\frac{3}{M(M+2)}\left(2|W|^{2}\left(v_{j}^{2}-w_{k}^{2}\right)+v_{j}^{4}+w_{k}^{4}-2 v_{j}^{2} w_{k}^{2}\right),
\end{aligned}
$$

and summing over $j$ and $k$ as before

$$
\begin{aligned}
{[\mathrm{UH}](V, W)=} & \frac{N}{M}\left(1-\frac{3}{M+2}\right) m_{4}(V)-\frac{N}{M}\left(1+\frac{3}{M+2}\right) m_{4}(W) \\
& -\frac{6}{M(M+2)}\left(1-\frac{1}{M}\right)|W|^{2}|V|^{2}+\frac{6 N}{M^{2}(M+2)}|W|^{4} .
\end{aligned}
$$

After some manipulations, one then gets an equation for $\zeta(t)$ :

$$
\begin{aligned}
\zeta(t) & =e^{-N \lambda \Delta_{M} t} \zeta(0) \\
& +\int_{0}^{t} e^{-N \lambda \Delta_{M}(t-s)}\left(\frac{N(M-1)}{M(M+2)} \xi(s)-\frac{N(M+5)}{M(M+2)} \zeta(s)\right. \\
& -\frac{6(M-1)\left(2 N-M^{2}-2 M\right)}{M(M+2)^{2}} \eta(s) \\
& \left.+\frac{3(N+2 M+4)(M-1)}{(M+2)^{2}} \psi(s)+\frac{3 N\left(4-3 M-M^{2}\right)}{M(2+M)^{2}}\right) d s .
\end{aligned}
$$


Differentiating these expressions, we find a linear system of differential equations for $\Psi(t)=$ $(\psi(t), \xi(t), \zeta(t))^{t}$

$$
\frac{d}{d t} \Psi(t)=\mathbf{A} \Psi(t)+\mathbf{b}_{1}+\mathbf{b}_{2} \eta(t)
$$

The initial values depend on the moments of the initial density $F_{N M}(V, W, 0)$, and are bounded by

$$
\begin{aligned}
|\eta(0)| & \leq N / M, \\
0 \leq \psi(0) & \leq(N / M)^{2}, \\
|\zeta(0)| & \leq N^{2} / M, \quad \text { and } \\
0 \leq \xi(0) & \leq 2 N .
\end{aligned}
$$

These bounds may be achieved, and hence the momentes are not bounded uniformly in $N$ and $M$ unless further hypothesis are made on intial data. As we shall see, this is not very critical for $\eta(t), \psi(t)$, and $\zeta(t)$, because after an initial interval of length $\sim M / N$, the transient part of the solution will be small for these variables. However, this is not the case for $\xi(t)$, and to conclude our proof of convergence to the BGK-equation, we shall have to assume the initial data $F_{N M}(V, W, 0)$ are such that $\xi(0) / M \rightarrow 0$ when $M \rightarrow \infty$.

We continue with an asymptotic analysis of the system of equations (66). The matrix $\mathbf{A}$ and vectors $\mathbf{b}_{1}$ and $\mathbf{b}_{1}$ are expressions involving $N$ and $M$. We have $\mathbf{A}=\mathbf{A}^{0}+\mathbf{A}^{r}$, where

$$
\mathbf{A}^{0}=\left(\begin{array}{ccc}
-2 \frac{N}{M} & \frac{N}{M^{2}} & \frac{N}{M^{2}} \\
3 \frac{N}{M} & -\frac{N}{M}-N \lambda \Delta_{M} & \frac{N}{M} \\
3 & 1 & -1
\end{array}\right),
$$

where in the third element of the third row, we have taken the exponential factor in (65) into account, and the components of $\mathbf{A}^{0}$ and $\mathbf{A}^{r}$ satisfy $\mathbf{a}^{r}{ }_{i j} / \mathbf{a}^{0}{ }_{i j}=\mathcal{O}(M / N+1 / M)$, or smaller, when $N, M \rightarrow \infty$. And similarly for the constant vector $\mathbf{b}=\mathbf{b}^{0}+\mathbf{b}^{r}$, we get

$$
\mathbf{b}_{1}^{0}=\left(\begin{array}{c}
\frac{N}{M^{2}} \\
-3 \frac{N}{M} \\
3
\end{array}\right) \quad \text { and } \quad \mathbf{b}_{2}^{0}=\left(\begin{array}{c}
4 \frac{N}{M^{2}} \\
6-12 \frac{N}{M^{2}} \\
6
\end{array}\right)
$$

The components of $\mathbf{b}^{r}$ are dominated by the components of $\mathbf{b}^{0}$ in the same way as the elements of $\mathbf{A}$. And because $\eta(t) \leq N M^{-1} e^{-\frac{N}{M} t}$, we see that for any fixed $t_{0}>0, \mathbf{b}_{2} \eta(t)$ will be negligible compared to $\mathbf{b}_{1}$, uniformly in $t>t_{0}$, when $N / M$ is sufficiently large. For large values of $N, M$, and $\lambda$, all but the last line of $\mathbf{A}^{0}$ will be dominated by the diagonal elements. The eigenvalues of $\mathbf{A}$ are asymptotically

$$
\begin{aligned}
& \ell_{1}=-2 \frac{N}{M}\left(1+\mathcal{O}\left(\frac{1}{M}\right)\right), \\
& \ell_{2}=-N \lambda \Delta_{M}\left(1+\mathcal{O}\left(\frac{1}{M}\right)\right) \quad \text { and } \\
& \ell_{3}=-1+\mathcal{O}\left(\frac{1}{M}\right),
\end{aligned}
$$

and we set $\ell_{0}=-(1+N / M)$, the decay rate of $\eta(t)$. The $\mathcal{O}$-terms also contain terms of the form $\mathcal{O}(1 / N)$ and $\mathcal{O}\left(1 / \lambda \Delta_{M}\right)$ but we will need $\lambda \Delta_{M}>>N>>M$, and therefore all remainder terms can be absorbed in one term that is $\mathcal{O}(1 / M)$. 
The solution to equation (66) is explicitly given by

$$
\left.\Psi(t)=\exp (t \mathbf{A}) \Psi(0)+\int_{0}^{t} \exp ((t-s) \mathbf{A})\right)\left(\mathbf{b}_{1}+\mathbf{b}_{2} \eta(s)\right) d s .
$$

We use Sylvester's formula to compute the exponential $\exp (t A)$ :

$$
e^{t \mathbf{A}}=\sum_{j=1}^{3} e^{\ell_{j} t} \mathbf{A}_{j}
$$

where

$$
\mathbf{A}_{j}=\prod_{k, k \neq j} \frac{1}{\ell_{j}-\ell_{k}}\left(\mathbf{A}-\ell_{k} I\right)
$$

Therefore

$$
\Psi(t)=\sum_{j=1}^{3}\left(e^{\ell_{j} t} \mathbf{A}_{j} \Psi(0)+\frac{1}{\ell_{j}}\left(1-e^{\ell_{j} t}\right) \mathbf{A}_{j} \mathbf{b}_{1}+\frac{1}{\ell_{0}-\ell_{j}}\left(e^{\ell_{0} t}-e^{\ell_{j} t}\right) \mathbf{A}_{j} \mathbf{b}_{2} \eta(0)\right),
$$

and this expression can be evaluated at least asymptotically as $N / M, M$, and $\lambda$ increase to infinity. For the purpose of this paper we need to prove that for any $t>0$ (and uniformly for $\left.t \geq t_{0}>0\right), \psi(t) \rightarrow 0$ when $N / M, M$, and $\lambda \rightarrow \infty$ as stated below, but all components of $\Psi(t)$ are needed to obtain a closed system.

The components of the matrices are all rational expressions of $N / M, M$, and $\lambda \Delta_{M}$, therefore all terms involving a $e^{\ell_{j} t}, j=0,1,2$ vanish when $M, N / M$ and $\lambda \Delta_{M}$ increase to infinity and for $t>0$, uniformly for $t \geq t_{0}>0$. It is therefore enough to study the terms that are constant in $t$ or with an exponential factor $e^{\ell_{3} t}$ (we recall that $\ell_{3} \sim-1$ in the limit of interest).

The matrices $\mathbf{A}_{j}, j=1,2,3$ are, asymptotically,

$$
\mathbf{A}_{1}=\left(\begin{array}{ccc}
1 & \frac{1}{M^{2} \lambda \Delta_{M}} & -\frac{1}{2 M} \\
\frac{3}{M \lambda \Delta_{M}} & -\frac{9}{4 M} & \frac{9}{4 M^{2} \lambda \Delta_{M}} \\
-\frac{3 M}{2 N} & \frac{3}{4 M N \Delta_{M}} & \frac{3}{4 N}
\end{array}\right) \quad \mathbf{A}_{2}=\left(\begin{array}{ccc}
\frac{3}{M^{3} \lambda^{2} \Delta_{M}^{2}} & -\frac{1}{M^{2} \lambda \Delta_{M}} & \frac{1}{M^{3} \lambda^{2} \Delta_{M}^{2}} \\
-\frac{3}{M \lambda \Delta_{M}} & 1 & -\frac{1}{M \Delta_{M}} \\
\frac{3}{M N \lambda^{2} \Delta_{M}^{2}} & -\frac{1}{N \lambda \Delta_{M}} & \frac{1}{M N \lambda^{2} \Delta_{M}^{2}}
\end{array}\right)
$$

and

$$
\mathbf{A}_{3}=\left(\begin{array}{ccc}
-\frac{3}{4 M^{2}} & -\frac{9}{4 M^{3} \lambda \Delta_{M}} & \frac{1}{2 M} \\
\frac{24}{M^{2}} & \frac{9}{4 M} & \frac{1}{M \lambda \Delta_{M}} \\
\frac{3 M}{2 N} & \frac{1}{N \lambda \Delta_{M}} & 1
\end{array}\right) .
$$

The constant terms, which also absorb the exponential factors which are related to the term $\mathbf{b}_{1}$ in Eq. (66), are $\frac{1}{\ell_{j}} \mathbf{A}_{j} \mathbf{b}_{1}$, and asymptotically these expressions have magnitudes

$$
\begin{aligned}
& j=1: \quad \frac{M}{2 N}\left(\frac{N}{M^{2}}+\frac{1}{M^{2} \lambda \Delta_{M}} \frac{3 N}{M}+3 \frac{1}{2 M}\right) \leq \frac{C}{M} \\
& j=2: \quad \frac{1}{N \lambda \Delta_{M}}\left(\frac{3}{M^{3} \lambda^{2} \Delta_{M}^{2}} \frac{N}{M^{2}}+\frac{1}{M^{2} \lambda \Delta_{M}} \frac{3 N}{M}+3 \frac{1}{M^{3} \lambda^{2} \Delta_{M}^{2}}\right) \leq \frac{C}{M^{3} \lambda^{2} \Delta_{M}^{2}} \\
& j=3: \quad \frac{3}{4 M^{2}} \frac{N}{M^{2}}+\frac{9}{4 M^{3} \lambda \Delta_{M}} \frac{3 N}{M}+3 \frac{1}{2 M} \leq C\left(\frac{N}{M^{4}}+\frac{1}{M}\right) .
\end{aligned}
$$


It remains to look at the exponential terms multiplying the initial data. The relevant term is bounded by

$$
\frac{3}{4 M^{2}} \psi(0)+\frac{9}{4 M^{3} \lambda \Delta_{M}} \zeta(0)+\frac{1}{2 M} \xi(0) .
$$

Already the constraints on the initial data stated in (67) imply that the three terms are bounded by a constant multiplied by

$$
\frac{N^{2}}{M^{4}}, \quad \frac{N^{2}}{M^{4} \lambda \Delta_{M}}, \quad \text { and } \quad \frac{N}{M},
$$

respectively, and imposing that $N / M^{2} \rightarrow 0$ when $N \rightarrow \infty$ the first two terms of (77) vanish in the limit. The condition that $N / M^{2} \rightarrow \infty$ may be relaxed to $N / M^{4} \rightarrow 0$ if instead we require $\psi(0) / M^{2} \rightarrow 0$ when $M \rightarrow 0$. And we do need to impose that $\xi(0) / M \rightarrow 0$ when $N, M \rightarrow \infty$.

Summarizing these estimates we obtain the following result:

Lemma 3 Let $\eta(t), \psi(t), \zeta(t)$, and $\xi(t)$ be moments of solutions $F_{N M}(V, W, t)$ to Eq. (7), as defined in Eq. (54). Directly from the definition it follows that $\eta, \psi, \zeta$, and $\xi$ are bounded by $N / M,(N / M)^{2}, N^{2} / M$, and $2 N$, respectively. Assume that $N, M$ and $\lambda$ increase to infinity in such a way that $N / M \rightarrow \infty, N / M^{2} \rightarrow 0$, and assume in addition that

$$
\frac{\xi(0)}{M}=\frac{1}{M} \int_{\Omega_{0,0}} F_{N M}(V, W, 0)\left(\frac{1}{M} \sum_{k=1}^{M} w_{k}^{4}\right) d \sigma \rightarrow 0 .
$$

Fix $t_{0}>0$. Then for $t \geq t_{0}$ there is a constant depending on $t_{0}$ such that

$$
\psi(t)=\int_{\Omega_{0,0}} F_{N M}(V, W, 0)(\tau(V)-1)^{2} d \sigma \leq C\left(\frac{N^{2}}{M^{4}}+\frac{\xi(0)}{M}\right)
$$

when $N, M$, and $\lambda$ go to infinity.

Remark 2 The lemma states that the mean energy per active particle converges to one when the number of particles increase as stated in the lemma. As presented here this is only certain for strictly positive times, but with further assumptions on the initial data $F_{N M}(V, W, 0)$ the same result could be achieved uniformly in time.

We are ready to prove the main result of this section, which says that when $N, M$, and $\lambda$ go to infinity as in the previous lemma, the first marginal of

$$
c(V)=\int_{\mathbb{R}}\left(\frac{1}{\sqrt{\tau\left(V_{w_{1}}^{j}\right)}} \mathcal{M}_{M}\left(\frac{v_{j}}{\sqrt{\tau\left(V_{w_{1}}^{j}\right)}}\right)-\mathcal{M}_{M}\left(v_{j}\right)\right) f_{N M}^{(N 0)}\left(V_{w_{1}}^{j}, t\right) d w_{1}
$$

from Eq. (28) converges to zero.

Lemma 4 For all bounded functions $g \in C(\mathbb{R})$,

$$
\left|\int_{\mathbb{R}^{N}} c(V) g\left(v_{1}\right) d V\right| \leq \sqrt{\psi(t)}
$$

and this converges to zero when $N, M$, and $\lambda$ increase to infinity according to Lemma 3. 
Proof Multiplying $c(V)$ with $g\left(v_{1}\right)$ and integrating gives

$$
\begin{aligned}
& \int_{\mathbb{R}^{N}} c(V) g\left(v_{1}\right) d V \\
& =\int_{\mathbb{R}^{N}} \int_{\mathbb{R}}\left(\frac{1}{\sqrt{\tau\left(V_{w_{1}}^{1}\right)}} \mathcal{M}_{M}\left(\frac{v_{1}}{\sqrt{\tau\left(V_{w_{1}}^{1}\right)}}\right)-\mathcal{M}_{M}\left(v_{j}\right)\right) f_{N M}^{(N 0)}\left(V_{w_{1}}^{1}, t\right) d w_{1} g\left(v_{1}\right) d V \\
& =\int_{\mathbb{R}^{N}} \int_{\mathbb{R}}\left(\frac{1}{\sqrt{\tau(V)}} \mathcal{M}_{M}\left(\frac{u}{\sqrt{\tau(V)}}\right)-\mathcal{M}_{M}(u)\right) g(u) d u f_{N M}^{(N 0)}(V, t) d V
\end{aligned}
$$

This integral may be estimated by noting that

$$
\begin{aligned}
\left|\frac{1}{s} \mathcal{M}_{M}\left(\frac{u}{s}\right)-\mathcal{M}_{M}(u)\right| \leq & \mathbb{1}_{\left|s^{2}-1\right| \geq \frac{1}{2}}\left(\frac{1}{s} \mathcal{M}_{M}\left(\frac{u}{s}\right)+\mathcal{M}_{M}(u)\right) \frac{|s-1|}{1 / 2} \\
& +\mathbb{1}_{|s-1|<\frac{1}{2}} \sup _{|\bar{s}-1|<\frac{1}{2}}\left|\frac{1}{\bar{s}^{2}} \mathcal{M}_{M}\left(\frac{u}{\bar{s}}\right)+\frac{u}{\bar{s}^{3}} \mathcal{M}_{M}^{\prime}\left(\frac{u}{\bar{s}}\right)\right||s-1|
\end{aligned}
$$

We have $\mathcal{M}_{M}(x) \rightarrow \frac{1}{\sqrt{2 \pi}} \exp \left(-x^{2} / 2\right)$ when $M \rightarrow \infty$ (see Eq. (24)), and similarly $\mathcal{M}_{M}^{\prime}(x) \rightarrow-\frac{x}{\sqrt{2 \pi}} \exp \left(-x^{2} / 2\right)$, and therefore that

$$
\left|\frac{1}{s} \mathcal{M}_{M}\left(\frac{u}{s}\right)-\mathcal{M}_{M}(u)\right| \leq\left(\frac{2}{s} e^{-(u / s)^{2} / 2}+2 e^{-u^{2} / 2}+32\left(1+u^{2}\right) e^{-u^{2} / 8}\right)|s-1|
$$

when $M$ is large enough. In Eq. (83) we may then estimate $g\left(v_{1}\right)$ with $\|g\|_{\infty}$, and then carry out the integral over $u$ to get

$$
\begin{aligned}
\left|\int_{\mathbb{R}^{N}} c(V) g\left(v_{1}\right) d V\right| & \leq C\|g\|_{\infty} \int_{\mathbb{R}^{N}} f_{N M}^{(N 0)}(V)|\sqrt{\tau(V)}-1| d V \\
& \left.\leq C\|g\|_{\infty} \int_{\mathbb{R}^{N}} f_{N M}^{(N 0)}(V) \mid \tau(V)\right)-1 \mid d V \\
& \left.\leq C\|g\|_{\infty}\left(\int_{\mathbb{R}^{N}} f_{N M}^{(N 0)}(V)(\tau(V))-1\right)^{2} d V\right)^{1 / 2}=C\|g\|_{\infty} \sqrt{\psi(t)}
\end{aligned}
$$

The constant $C$ here is obtained by integrating the expression (84). Then Lemma 3 provides the needed bounds for $\psi(t)$

\section{Proof and Conclusions}

The goal of this section is to prove Theorem 1. Recall from Eq. (28) that the equation for the $(N, 0)$-marginals can be written

$$
\frac{\partial}{\partial t} f_{N M}^{(N 0)}(V, t)=a(V)+b(V)+c(V)+I_{2}(V)
$$


and hence that, for any bounded, continuous function $g\left(v_{1}\right)$,

$$
\begin{aligned}
\frac{\partial}{\partial t} \int_{|V|^{2} \leq N+M} f_{N M}^{(N 0)}(V, t) g\left(v_{1}\right) d V & =\frac{\partial}{\partial t} \int_{-\sqrt{N+M}}^{\sqrt{N+M}} f_{N M}^{(10)}\left(v_{1}, t\right) g\left(v_{1}\right) d v_{1} \\
& =\int_{|V|^{2} \leq N+M} a(V) g\left(v_{1}\right) d V+\int_{|V|^{2} \leq N+M} b(V) g\left(v_{1}\right) d V \\
& +\int_{|V|^{2} \leq N+M} c(V) g\left(v_{1}\right) d V a+\int_{|V|^{2} \leq N+M} I_{2}(V) g\left(v_{1}\right) d V,
\end{aligned}
$$

These terms have been analysed above in this paper, and it only remains to put the pieces together. The first term, the integral of $a(V)$,

$$
\int_{|V|^{2} \leq N+M} a(V) g\left(v_{1}\right) d V=\int_{v_{1}^{2} \leq N+M}\left(\mathcal{M}\left(v_{1}\right)-f_{N, M}^{1,0}\left(v_{1}\right)\right) g\left(v_{1}\right) d v_{1} .
$$

converges to the righthand side of equation (14) (see Eq. (29)),

The second term, the integral of $b(V)$, converges to zero because $\mathcal{M}_{M}(w)$ converges pointwise to the Maxwellian $\mathcal{M}$ (see Eq. (30)).

That the third term converges to zero is exactly the content of Lemma 4, and finally Lemma 2 states that

$$
\int_{|V|^{2} \leq N+M} I_{2}(V) g\left(v_{1}\right) d V
$$

converges to zero if $\left\|F_{N M}(\cdot, \cdot, 0)\right\|_{L^{2}\left(\Omega_{0,0}\right)} / \lambda \rightarrow 0$. By hypothesis (see Eq. (13)), $\| F_{N M}$ $(\cdot, \cdot, 0) \|_{L^{2}\left(\Omega_{0,0}\right)} \leq C_{N M}$ for a family of constansts $C_{N M}$ and we may the choose $\lambda=\lambda_{N M}$ accordingly. .

Now let $f(v, t)$ be the solution of

$$
\partial_{t} f(v, t)=\mathcal{M}-f(v, t) .
$$

Then

$$
\begin{aligned}
\frac{\partial}{\partial t} \int_{\mathbb{R}} g\left(v_{1}\right)\left(f_{N M}^{(10)}\left(v_{1}, t\right)-f\left(v_{1}, t\right)\right) d v_{1} & =-\int_{\mathbb{R}} g\left(v_{1}\right)\left(f_{N M}^{(10)}\left(v_{1}, t\right)-f\left(v_{1}, t\right)\right) d v_{1} \\
& +\int_{|V|^{2} \leq N+M} b(V) g\left(v_{1}\right) d V \\
& +\int_{|V|^{2} \leq N+M} c(V) g\left(v_{1}\right) d V \\
& +\int_{|V|^{2} \leq N+M} I_{2}(V) g\left(v_{1}\right) d V,
\end{aligned}
$$

and it follows that

$$
\int_{\mathbb{R}} g\left(v_{1}\right)\left(f_{N M}^{(10)}\left(v_{1}, t\right)-f\left(v_{1}, t\right)\right) d v_{1}
$$

converges to zero under the assumptions of the theorem.

This concludes the proof of Theorem 1. 
Acknowledgements Open access funding provided by Chalmers University of Technology. We would like to thank Pierre Degond for helpful discussions. D.M. acknowledges support by the Swedish Science Council. B.W. acknowledges support by the Swedish Science Council, the Knut and Alice Wallenberg foundation and the Swedish Foundation for Strategic Research.

Open Access This article is licensed under a Creative Commons Attribution 4.0 International License, which permits use, sharing, adaptation, distribution and reproduction in any medium or format, as long as you give appropriate credit to the original author(s) and the source, provide a link to the Creative Commons licence, and indicate if changes were made. The images or other third party material in this article are included in the article's Creative Commons licence, unless indicated otherwise in a credit line to the material. If material is not included in the article's Creative Commons licence and your intended use is not permitted by statutory regulation or exceeds the permitted use, you will need to obtain permission directly from the copyright holder. To view a copy of this licence, visit http://creativecommons.org/licenses/by/4.0/.

\section{References}

1. Andries, P., Aoki, K., Perthame, B.: A consistent BGK-type model for gas mixtures. J. Stat. Phys. 106(56), 993-1018 (2002)

2. Asadzadeh, M., Kazemi, E., Mokhtari, R.: Discrete-ordinates and streamline diffusion methods for a flow described by BGK model. SIAM J. Sci. Comput. 36(4), B729-B748 (2014)

3. Bhatnagar, P.L., Gross, E.P., Krook, M.: A model for collision processes in gases. I. Small amplitude processes in charged and neutral one-component systems. Phys. Rev. 94(3), 511-525 (1954)

4. Bisi, M., Cáceres, M.J.: A BGK relaxation model for polyatomic gas mixtures. Commun. Math. Sci. 14(2), 297-325 (2016)

5. Bonetto, F., Loss, M., Tossounian, H., Vaidyanathan, R.: Uniform approximation of a Maxwellian thermostat by finite reservoirs. Commun. Math. Phys. 351(1), 311-339 (2017)

6. Bonetto, F., Loss, M., Vaidyanathan, R.: The Kac model coupled to a thermostat. J. Stat. Phys. 156(4), 647-667 (2014)

7. Buttà, P., Hauray, M., Pulvirenti, M.: Particle approximation of the BGK equation, arXiv:2002.10535 [math-ph] (2020)

8. Carlen, E.A., Carvalho, M.C., Loss, M.: Determination of the spectral gap for Kac's master equation and related stochastic evolution. Acta Math. 191(1), 1-54 (2003)

9. Chen, H., Orszag, S.A., Staroselsky, I.: Macroscopic description of arbitrary knudsen number flow using boltzmann-bgk kinetic theory. J. Fluid Mech. 574, 495-505 (2007)

10. Colosqui, C.E.: High-order hydrodynamics via lattice Boltzmann methods. Phys. Rev. E 81(2), 026702 (2010)

11. Gallagher, I., Saint-Raymond, L., Texier, B.: From Newton to Boltzmann: Hard Spheres and Short-Range Potentials. Zurich Lectures in Advanced Mathematics. European Mathematical Society (EMS), Zürich (2013)

12. Groppi, M., Spiga, G.: An ES-BGK model for the kinetic analysis of a chemically reacting gas mixture. MATCH Commun. Math. Comput. Chem. 69(2), 197-214 (2013)

13. Groppi, M., Russo, G., Stracquadanio, G.: High order semi-Lagrangian methods for the BGK equation. Commun. Math. Sci. 14(2), 389-414 (2016)

14. Henin, F.: Asymptotic evolution in Kac's model. Acad. Roy. Belg. Bull. Cl. Sci. 5(60), 686-720 (1974)

15. Janvresse, E.: Spectral gap for Kac's model of the Boltzmann equation. Ann. Probab. 29(1), 288-304 (2001)

16. Jung, J., Wennberg, B.: The BBGKY hierarchy and the BGK equation. Unpublished

17. Kac, M.: Probability and Related Topics in Physical Sciences, volume 1957 of With Special Lectures by G. E. Uhlenbeck, A. R. Hibbs, and B. van der Pol. Lectures in Applied Mathematics. Proceedings of the Summer Seminar, Boulder, Colo. Interscience Publishers, London-New York (1959)

18. Lanford, III, O.E.: Time evolution of large classical systems. In: Dynamical Systems, Theory and Applications (Recontres, Battelle Res. Inst., Seattle, Wash., 1974)

19. Mustafa, D.: Propagation of Chaos for Kac-like Particle Systems. Doktorsavhandlingar vid Chalmers tekniska högskola. Ny serie, no: 3976. Department of Mathematical Sciences, Chalmers University of Technology (2015)

20. Perthame, B.: Global existence to the BGK model of Boltzmann equation. J. Differ. Equ. 82(1), 191-205 (1989) 
21. Perthame, B., Pulvirenti, M.: Weighted $L^{\infty}$ bounds and uniqueness for the Boltzmann BGK model. Arch. Ration. Mech. Anal. 125(3), 289-295 (1993)

22. Saint-Raymond, L.: Discrete time Navier-Stokes limit for the BGK Boltzmann equation. Commun. Partial Differ. Eq. 27(1-2), 149-184 (2002)

23. Simons, S.: The nonlinear BGK model-a derivation and two applications. J. Phys. A 5(10), 1537-1544 (1972)

24. Tossounian, H., Vaidyanathan, R.: Partially thermostated Kac model. J. Math. Phys. 56(8), 083301 (2015)

25. Welander, P.: On the temperature jump in a rarefied gas. Arkiv Fysik 7, 507-553 (1954)

26. Xuan, L.-J., Kun, X.: A new gas-kinetic scheme based on analytical solutions of the BGK equation. J. Comput. Phys. 234, 524-539 (2013)

27. Yun, S.-B.: Cauchy problem for the Boltzmann-BGK model near a global Maxwellian. J. Math. Phys. 51(12), 123514 (2010)

28. Yun, S.-B.: Ellipsoidal BGK model near a global Maxwellian. SIAM J. Math. Anal. 47(3), 2324-2354 (2015)

Publisher's Note Springer Nature remains neutral with regard to jurisdictional claims in published maps and institutional affiliations. 